\title{
Superior Parietal Cortex Is Critical for the Manipulation of Information in Working Memory
}

\author{
Michael Koenigs, ${ }^{1}$ Aron K. Barbey, ${ }^{2,3}$ Bradley R. Postle, ${ }^{1,4}$ and Jordan Grafman ${ }^{2}$ \\ ${ }^{1}$ Department of Psychiatry, University of Wisconsin-Madison, Madison, Wisconsin 53719, ${ }^{2}$ Cognitive Neuroscience Section, National Institute of \\ Neurological Disorders and Stroke, National Institutes of Health, Bethesda, Maryland 20892, 'Department of Psychology, Georgetown University, \\ Washington, DC 20057, and ${ }^{4}$ Department of Psychology, University of Wisconsin-Madison, Madison, Wisconsin 53706
}

In recent years, theoretical perspectives on posterior parietal function have evolved beyond the traditional visuospatial processing models to include more diverse cognitive operations, such as long-term and working memory. However, definitive neuropsychological evidence supporting the superior parietal lobule's purported role in working memory has been lacking. Here, we studied human brain lesion patients to determine whether the superior parietal lobule is indeed necessary for working memory. We assessed a wide range of memory functions in three participant groups: superior parietal lesions $(n=19)$, lesions not involving superior parietal cortex $(n=146)$, and no brain lesions $(n=55)$. Superior parietal damage was reliably associated with deficits on tests involving the manipulation and rearrangement of information in working memory, but not on working memory tests requiring only rehearsal and retrieval processes, nor on tests of long-term memory. These results indicate that superior parietal cortex is critically important for the manipulation of information in working memory.

\section{Introduction}

For decades, research on the function of superior aspects of the posterior parietal cortex (PPC) largely focused on visuospatial and attentional processing (Critchley, 1953; Colby and Goldberg, 1999; Nachev and Husain, 2006; Sack, 2009; Vandenberghe and Gillebert, 2009). More recently, this classic conception has been supplemented by a host of functional neuroimaging studies that reliably associate posterior parietal cortex activity with tasks involving long-term and working memory (Wager and Smith, 2003; Wagner et al., 2005; Cabeza et al., 2008). Despite the consistently observed correlation between activity in the superior parietal lobule (SPL) of the PPC and working memory task performance (Wager and Smith, 2003), there is sparse neuropsychological evidence to corroborate the importance of SPL in working memory. Of the neurological patient studies that have addressed the role of PPC in working memory (Warrington et al., 1971; Saffran and Marin, 1975; Husain et al., 2001; Pisella et al., 2004; Malhotra et al., 2005; Ravizza et al., 2005; Baldo and Dronkers, 2006; van Asselen et al., 2006; Berryhill and Olson, 2008a,b), all share one or more of the following features: small number of cases with PPC damage, lesions primarily involving inferior (rather than superior) PPC, and exclu-

\footnotetext{
Received July 30, 2009; revised Sept. 22, 2009; accepted 0ct. 22, 2009

This work was supported by funding from the U.S. National Institute of Neurological Disorders and Stroke intramural research program and a project grant from the United States Army Medical Research and Material Command administered by the Henry M. Jackson Foundation (Vietnam Head Injury Study Phase III: a 30-year post-injury follow-up study, grant number DAMD17-01-1-0675). We thank V. Raymont for assistance with patient testing and lesion tracing, and S. Bonifant for data management.

Correspondence should be addressed to either of the following: Michael Koenigs, Department of Psychiatry, University of Wisconsin-Madison, 6001 Research Park Boulevard, Madison, WI 53719, E-mail: mrkoenigs@wisc.edu; or Jordan Grafman, Cognitive Neuroscience Section, National Institute of Neurological Disorders and Stroke, National Institutes of Health, 10 Center Drive, Bethesda, MD 20892, E-mail: grafmanj@ninds.nih.gov.

DOI:10.1523/JNEUROSCI.3706-09.2009

Copyright $\odot 2009$ Society for Neuroscience $\quad$ 0270-6474/09/2914980-07\$15.00/0
}

sive use of visual-spatial or auditory-verbal working memory tests. In other words, there has been no comprehensive evaluation of working memory function in a relatively large group of patients with damage specifically involving superior parietal cortex, and across a broad range of tasks and stimulus material. The absence of such data represents a substantial gap in the understanding of both parietal lobe function and the neural substrates of working memory. Here, we characterize working memory function in a group of patients with focal brain lesions involving superior parietal cortex.

\section{Materials and Methods}

Participant data

We drew participants from the Phase 3 Vietnam Head Injury Study (VHIS) registry, which includes American veterans who suffered brain damage from penetrating head injuries in the Vietnam War $(n=199)$, as well as neurologically healthy Vietnam veterans $(n=55)$. To preclude the possibility that defective performance on working memory tests could be secondary to deficits in the production and/or comprehension of language, rather than due to a primary deficit in working memory per se, we excluded any participant who had significant impairment (defined as performance at least two SDs below the mean of the neurologically healthy group) on tests of language production and language comprehension (supplemental Table 1, available at www.jneurosci.org as supplemental material). From the remaining brain-injured veterans we selected those with significant damage to SPL in either hemisphere (SupPar Lesion group; $n=19$ ) (Fig. 1; supplemental Fig. 1, available at www. jneurosci.org as supplemental material), as well as a comparison group of brain-injured veterans whose damage did not include SPL in either hemisphere (NonSupPar Lesion group; $n=146$ ). The neurologically healthy veterans (No Lesion group; $n=55$ ) served as an additional comparison group. Demographic and background cognitive function data for the three groups are presented in Table 1.

\section{Lesion analysis}

We acquired computed tomography (CT) data during the Phase 3 testing period. Magnetic resonance imaging was contraindicated by the history 

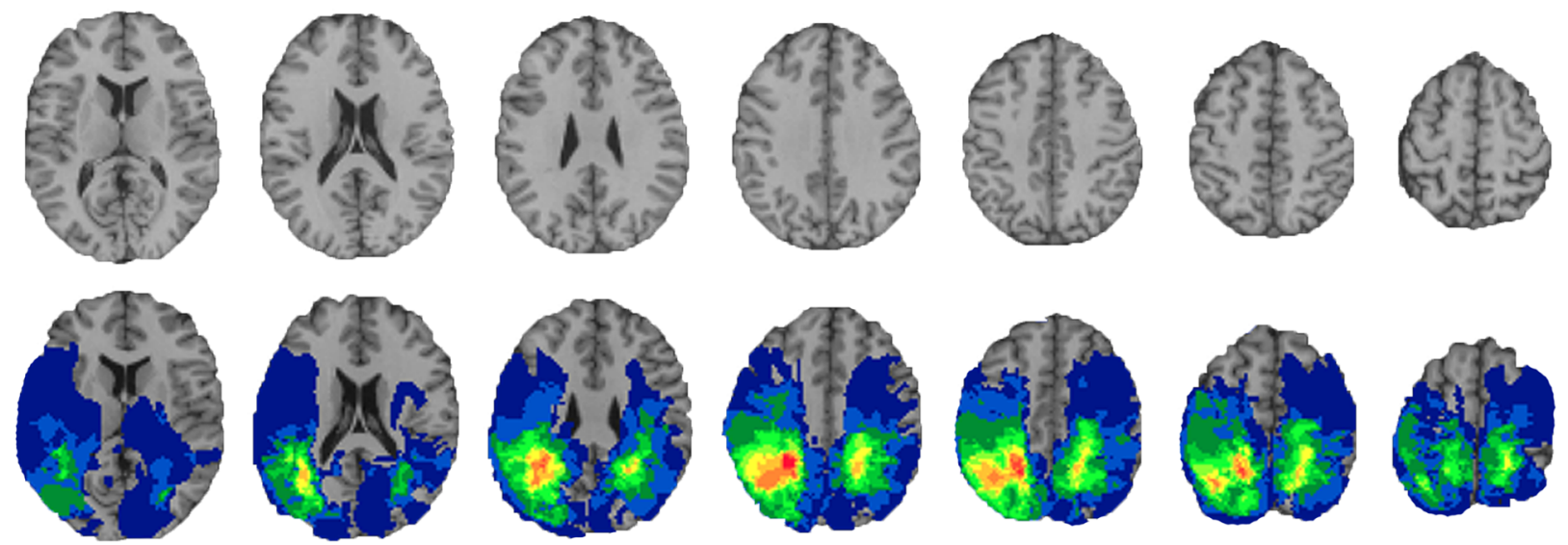

Figure 1. Lesion overlap of the SupPar Lesion group. Top row, Transverse slices of a normal healthy adult brain, for reference. Bottom row, Color indicates the number of overlapping lesions at each voxel. Maximal overlap in each hemisphere occurs superior and medial to the intraparietal sulcus, in the superior parietal lobe. In each slice the right hemisphere is on the reader's left.

Table 1. Demographic and background data

\begin{tabular}{llllllll}
\hline Group & Age & Sex (\% male) & Yrs Edu & Pre-combat AFQT & Lesion size $\left(\mathrm{cm}^{3}\right)$ & Verbal Comp & Boston Naming \\
\hline No Lesion & $59.0(3.4)$ & 100 & $15.2(2.5)$ & $65.4(22.9)$ & n/a & $109.7(12.0)$ & $55.4(4.7)$ \\
NonSupPar Lesions & $58.3(3.3)$ & 100 & $14.8(2.5)$ & $61.5(25.1)$ & $32.0(32.0)$ & $107.7(14.2)$ & $54.9(4.1)$ \\
SupPar Lesions & $57.9(2.2)$ & 100 & $14.5(2.2)$ & $61.7(24.2)$ & $62.6(47.9)$ & $107.3(11.1)$ & $57.9(2.2)$ \\
\hline
\end{tabular}

Data are presented as means with SDs in parentheses. "Age" refers to age at the time of Phase 3 evaluation. "Yrs Edu" refers to years of education. "Pre-combat AFQT" refers to percentile scores on the Armed Forces Qualification Test, a battery of tests measuring basic cognitive function at the time of enlistment (pre-injury). "Verbal Comp" refers to the Phase 3 Verbal Comprehension Index score from the Wechsler Adult Intelligence Scale. "Boston Naming" refers to raw scores from the Phase 3 Boston Naming Test. There were no significant differences among groups for any measure except lesion size.

of penetrating metal fragments in the patient sample. We acquired Axial CT scans without contrast at Bethesda Naval Hospital on a GE Medical Systems Light Speed Plus CT scanner in helical mode. We reconstructed the images with an in-plane voxel size of $0.4 \times 0.4 \mathrm{~mm}$, overlapping slice thickness of $2.5 \mathrm{~mm}$ and a $1 \mathrm{~mm}$ slice interval. We determined lesion location and volume from CT images using the Analysis of Brain Lesion (ABLe) software (Makale et al., 2002; Solomon et al., 2007) contained in MEDx v3.44 (Medical Numerics) with enhancements to support the Automated Anatomical Labeling (AAL) atlas (Tzourio-Mazoyer et al., 2002). As part of this process, we spatially normalized the CT image of each subject's brain to a CT Template brain image in MNI (Montreal Neurological Institute) space (Collins et al., 1994). We calculated lesion volume by manual tracing of the lesion in all relevant slices of the CT image then summing the traced areas and multiplying by slice thickness. A trained neuropsychiatrist performed the manual tracing, which was then reviewed by J.G., who was blind to the results of the neuropsychological testing. The superior parietal region of interest was defined as the area of parietal lobe (including gray and white matter) superior and medial to the intraparietal sulcus (IPS).

\section{Neuropsychological tests}

Language production and comprehension. In the Boston Naming Test (Goodglass and Kaplan, 2000), the subject views a series of 60 ink drawings of objects and attempts to name aloud each item. In the Token Test (Boller and Vignolo, 1966), the subject attempts to follow a series of 62 oral commands (e.g., "Touch the green square and the blue circle"). The Verbal Comprehension Index is a composite of scores from the Information, Similarities, and Vocabulary tests from the Wechsler Adult Intelligence Scale-III (Wechsler, 1997a).

Working memory. In Digit Span Forward (Wechsler, 1997a), the subject hears a sequence of digits and attempts to repeat the sequence in order. Sequence length is increased until the subject can no longer correctly repeat the sequence. Digit Span Backward (Wechsler, 1997a) is the same as Digit Span Forward, except the subject attempts to repeat the sequence in reverse order. In Spatial Span Forward (Wechsler, 1997a), the subject watches the examiner tap a sequence of locations on a board and attempts to repeat the tapping sequence in order. Sequence length is increased until the subject can no longer correctly repeat the sequence.
Spatial Span Backward (Wechsler, 1997a) is the same as Spatial Span Forward, except the subject attempts to repeat the sequence in reverse order. In Letter-Number Sequencing (Wechsler, 1997a), the subject hears a sequence of alternating digits and letters and attempts to repeat the digits and letters from the sequence, beginning with the digits in numerical order, followed by the letters in alphabetical order. In Arithmetic (Wechsler, 1997a), the subject hears numerical problems in story format, performs mental arithmetic (i.e., without paper and pencil), and makes a verbal response. In the N-Back test, the subject hears a series of digits, and for each new digit, reports whether the new digit is the same as the digit " $n$ " steps back.

Long-term memory. In Logical Memory (Wechsler, 1997b), the subject hears two brief stories, and after each story immediately attempts to recall the story (immediate recall). After $30 \mathrm{~min}$, the subject attempts a second recall for each story (delayed recall). In Faces (Wechsler, 1997b), the subject views a series of 24 pictures of faces and then immediately thereafter a series of 48 face pictures including the 24 previously seen targets interspersed among 24 foils, and indicates whether or not each face was previously seen (immediate recognition). After $30 \mathrm{~min}$ a second recognition test is performed with 24 new foils (delayed recognition). In Verbal-Paired Associates (Wechsler, 1997b), the subject hears eight word-pairs. In the test portion the first word of the pair is presented, and the subject attempts to recall the second word of the pair. The test is administered immediately after the word-pair presentations (immediate recall) and 30 min later (delayed recall). In Family Pictures (Wechsler, 1997b), the subject views a picture of six family members, and then views individual pictures that contain four of the family members interacting in various situations. Subjects attempt to report on the identity, location, and action of each character in each picture, once immediately after the picture is removed (immediate recall) and once after $30 \mathrm{~min}$ (delayed recall).

Visuo-spatial rearrangement. In the Tower Test (Delis et al., 2001), subjects attempt to rearrange discs on a set of pegs to match a model. In Block Design (Wechsler, 1997a), subjects attempt to rearrange multicolored blocks to match a model design. In Object Assembly (Wechsler, 1997a), subjects attempt to rearrange puzzle pieces to form familiar objects. 
Table 2. Working memory test data

\begin{tabular}{|c|c|c|c|c|c|c|}
\hline & No Lesion & NonSupPar Lesions & SupPar Lesions & ANOVA Fvalue & ANOVA $p$ value & Significant between-group differences \\
\hline Digit Span Forward & $6.7(1.2)$ & $6.3(1.2)$ & $6.2(1.2)$ & 1.8 & 0.16 & None \\
\hline Digit Span Backward & $5.0(1.4)$ & $4.6(1.3)$ & $3.7(0.8)$ & 6.8 & 0.001 & $\begin{array}{l}\text { SupPar }<\text { No Lesion } \\
\text { SupPar }<\text { NonSupPar }\end{array}$ \\
\hline Spatial Span Forward & $8.0(1.5)$ & $7.6(1.7)$ & $7.0(2.0)$ & 2.8 & 0.07 & None \\
\hline Spatial Span Backward & $7.7(1.8)$ & $7.2(1.7)$ & $6.0(2.2)$ & 5.7 & 0.004 & $\begin{array}{l}\text { SupPar }<\text { No Lesion } \\
\text { SupPar }<\text { NonSupPar }\end{array}$ \\
\hline Letter-Number Sequencing & $10.2(2.4)$ & $9.4(2.3)$ & $7.8(2.0)$ & 7.5 & 0.001 & $\begin{array}{l}\text { SupPar }<\text { No Lesion } \\
\text { SupPar }<\text { NonSupPar }\end{array}$ \\
\hline Arithmetic & $15.0(3.3)$ & $13.7(3.6)$ & $10.1(3.7)$ & 13.7 & $<0.001$ & $\begin{array}{l}\text { SupPar }<\text { No Lesion } \\
\text { SupPar }<\text { NonSupPar }\end{array}$ \\
\hline 0-Back errors & $1.7(3.8)$ & $2.1(2.9)$ & $1.9(2.5)$ & 0.2 & 0.79 & None \\
\hline 1-Back errors & $4.4(5.4)$ & $5.2(4.0)$ & $5.8(3.7)$ & 0.8 & 0.44 & None \\
\hline 2-Back errors & $7.9(4.4)$ & $8.9(4.0)$ & $10.7(3.0)$ & 3.5 & 0.03 & SupPar $>$ No Lesion \\
\hline 3-Back errors & $9.6(3.6)$ & $10.9(4.0)$ & $12.2(4.2)$ & 3.4 & 0.03 & SupPar $>$ No Lesion \\
\hline
\end{tabular}

Means are presented with SDs in parentheses. One-way ANOVAs were conducted for each working memory test. Significant between-group differences were determined with Tukey's honestly significant difference test.

\section{Results}

\section{Working memory function}

To determine the effect of SPL lesions on working memory performance, we analyzed data from several canonical tests of working memory: Digit Span Forward, Digit Span Backward, Spatial Span Forward, Spatial Span Backward, Letter-Number Sequencing, Arithmetic, and N-Back (Table 2, supplemental Figs. 2-7, available at www.jneurosci.org as supplemental material).

To summarize the results reported in Table 2 and supplemental Figure 2-7 (available at www.jneurosci.org as supplemental material), there were no significant group differences for the tests that required sustained attention but minimal working memory demands (0-Back and 1-Back), or tests that required the simple retention and recall of information from working memory (Digit Span Forward and Spatial Span Forward), regardless of whether the stimuli were auditory-verbal (Digit Span Forward) or visualspatial (Spatial Span Forward). However, there were significant group differences for those tests that required the additional manipulation or rearrangement of information within working memory (Digit Span Backward, Spatial Span Backward, LetterNumber Sequencing, and Arithmetic), as well as for the more demanding working memory conditions of the N-Back test (2-Back and 3-Back). For all working memory tests that revealed a significant difference among groups, the SupPar Lesion group's performance was significantly worse than one or both comparison groups. The NonSupPar Lesion group's performance was not significantly different than the No Lesion group on any of the tests. These data suggest that SPL damage specifically impairs the manipulation and rearrangement of information in working memory. However, to substantiate this conclusion, it is necessary to examine several factors that are relevant to the interpretation of the observed results: (1) lesion size, (2) damage to areas adjacent to SPL (in particular, inferior parietal lobule), (3) lesion laterality, and (4) specificity of the cognitive deficit.

\section{Lesion size}

As can be seen in Table 1, the mean lesion size of the SupPar Lesion group is significantly greater than the NonSupPar Lesion group. Thus, the SupPar Lesion group's relatively poor performance on six of the working memory tests (Digit Span Backward, Spatial Span Backward, Letter-Number Sequencing, Arithmetic, 2-back, and 3-back) could conceivably be due to a difference in lesion size, rather than a difference in lesion location. We ad- dressed this possibility with two analyses. In the first analysis, we determined the correlations between lesion size and performance on the six key tests. For the NonSupPar Lesion group, the absolute values of all Pearson's $r$ values were 0.07 or less, and the corresponding $p$ values were 0.45 or greater (supplemental Table 2 , available at www.jneurosci.org as supplemental material). For the SupPar Lesion group, the absolute values of all Pearson's $r$ values were 0.26 or less, and the corresponding $p$ values were 0.29 or greater (supplemental Table 3, available at www.jneurosci.org as supplemental material). Thus, in both brain-injured patient groups, lesion size had no significant correlation with performance on any of the six tests. In the second analysis, we selected the subset of patients from the NonSupPar Lesion group with the largest lesions $(n=54)$, such that the mean lesion size in this NonSupPar Lesion "large lesion" subset $\left(62.7 \mathrm{~cm}^{3}\right)$ was nearly identical to the mean lesion size in the SupPar Lesion group (62.6 $\mathrm{cm}^{3}$ ). We then compared these two groups' performance on each of the six key tests (supplemental Table 4, available at www. jneurosci.org as supplemental material). Mirroring the main analysis (Table 2), the SupPar Lesion group was significantly worse than the NonSupPar Lesion large lesion subset on Digit Span Backward ( $p=0.004)$, Letter-Number Sequencing ( $p=$ 0.02 ), and Arithmetic ( $p=0.001)$, and nearly so on Spatial Span Backward $(p=0.05)$ and 2-back errors $(p=0.08)$. Together, these two analyses converge to indicate that lesion size, in and of itself, does not account for the observed pattern of results.

\section{Involvement of inferior parietal cortex}

As can be seen in Figure 1, some of the lesions in the SupPar Lesion group extend beyond the boundaries of SPL. In most areas of the brain outside SPL, the number of overlapping lesions is small $(0,1$, or 2$)$. However, right inferior parietal cortex is an exception; approximately half the patients in SupPar Lesion group have damage involving this area. Thus, the impaired performance of the SupPar Lesion group could conceivably be due to the damage to right inferior parietal cortex, rather than to SPL. To address this possibility, we selected two groups for a follow-up analysis (Fig. 2). From the NonSupPar Lesion group we selected those patients with damage that primarily involved right inferior parietal cortex (with minimal superior parietal involvement; $n=$ 6 ), and from the SupPar Lesion group we selected those patients with damage that primarily involved SPL (with minimal inferior parietal involvement; $n=6$ ). Mean lesion size was similar between these two groups $\left(35.1 \mathrm{~cm}^{3}\right.$ for the right inferior group, $40.1 \mathrm{~cm}^{3}$ for the superior group; $t=0.3 ; p=0.79$ ). We then 

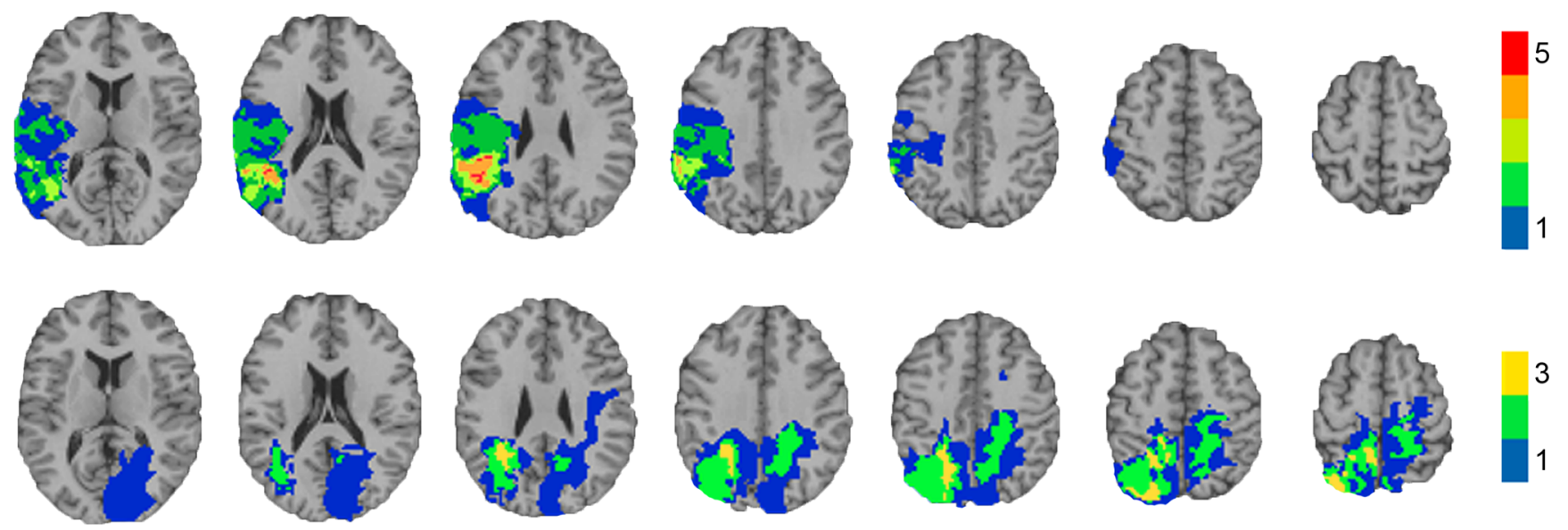

Figure 2. Lesion overlaps comparing right inferior parietal and superior parietal lesions. Color indicates the number of overlapping lesions at each voxel. In each slice the right hemisphere is on the reader's left. Top row, Lesion overlap for the right inferior parietal lesion group $(n=6)$. Lesions are lateral and inferior to the intraparietal sulcus. Bottom row, Lesion overlap for the focal superior parietal lesion group $(n=6)$. Lesions are primarily superior and medial to the intraparietal sulcus.

Table 3. Working memory test data for right inferior parietal and focal superior parietal lesion groups

\begin{tabular}{|c|c|c|c|c|c|c|}
\hline & No Lesion & Right InfPar Lesions & Focal SupPar Lesions & Kruskal-Wallis $\chi^{2}$ & $p$ value & Significant between-group differences \\
\hline Digit Span Forward & $6.7(1.2)$ & $7.0(1.4)$ & $6.0(1.1)$ & 2.3 & 0.32 & None \\
\hline Digit Span Backward & $5.0(1.4)$ & $5.5(1.0)$ & $3.3(0.8)$ & 8.9 & 0.01 & $\begin{array}{l}\text { SupPar }<\text { No Lesion } \\
\text { SupPar }<\text { RightInfPar }\end{array}$ \\
\hline Spatial Span Forward & $8.0(1.5)$ & $9.3(1.2)$ & $6.3(2.9)$ & 5.9 & 0.05 & None \\
\hline Spatial Span Backward & $7.7(1.8)$ & $8.8(0.8)$ & $6.7(2.6)$ & 3.5 & 0.17 & None \\
\hline Letter-Number Sequencing & $10.2(2.4)$ & $11.0(2.3)$ & $7.7(1.0)$ & 8.8 & 0.01 & $\begin{array}{l}\text { SupPar }<\text { No Lesion } \\
\text { SupPar }<\text { RightInfPar }\end{array}$ \\
\hline Arithmetic & $15.0(3.3)$ & $16.3(1.5)$ & $10.5(2.7)$ & 9.7 & 0.008 & $\begin{array}{l}\text { SupPar }<\text { No Lesion } \\
\text { SupPar }<\text { RightInfPar }\end{array}$ \\
\hline 0-Back errors & $1.7(3.8)$ & $0.3(0.8)$ & $2.3(3.0)$ & 3.1 & 0.22 & None \\
\hline 1-Back errors & $4.4(5.4)$ & $3.8(2.9)$ & $6.5(5.0)$ & 1.7 & 0.44 & None \\
\hline 2-Back errors & $7.9(4.4)$ & $5.2(3.8)$ & $11.3(3.5)$ & 6.9 & 0.03 & $\begin{array}{l}\text { SupPar }>\text { No Lesion } \\
\text { SupPar }>\text { RightInfPar }\end{array}$ \\
\hline 3-Back errors & $9.6(3.6)$ & $8.2(3.0)$ & $12.0(3.6)$ & 3.2 & 0.20 & None \\
\hline
\end{tabular}

Means are presented with SDs in parentheses. Because of the small sample sizes, nonparametric tests were used to test for group effects and for the pairwise comparisons. Significant between-group differences were determined with the Mann-Whitney $U$ test.

compared the performance of these two groups, along with the No Lesion group, to determine whether focal lesions to either right inferior parietal cortex or SPL would result in working memory impairment (Table 3 ). The group of patients with focal right inferior parietal lesions exhibited no significant differences from the No Lesion group on any of the working memory measures. The group of patients with focal superior parietal lesions, on the other hand, exhibited significantly worse performance than the No Lesion group on Digit Span Backward ( $p=0.007)$, Letter-Number Sequencing ( $p=0.006)$, Arithmetic $(p=0.005)$ and 2-back ( $p=0.03$ ), as well as significantly worse performance than the patients with focal right inferior parietal lesions on Digit Span Backward ( $p=0.007)$, Letter-Number Sequencing $(p=$ $0.006)$, Arithmetic $(p=0.005)$, and 2-back $(p=0.02)$. In addition, we examined the performance of those individuals with lesions involving both superior and inferior parietal areas $(n=$ 11; supplemental Table 5, available at www.jneurosci.org as supplemental material). As expected, this group of inferior+ superior parietal lesions performed significantly worse than the right inferior parietal lesion group on all working memory manipulation tests (Digit Span Backward $p=0.006$; Spatial Span Backward $p=0.005$; Letter-Number Sequencing $p=0.03$; Arithmetic $p=0.005 ; 2$-back $p=0.009 ; 3$-back $p=0.03)$, but similarly to the group of focal superior lesions (all $p$ values $>0.30$ ). These data indicate that superior parietal damage, but not right inferior parietal damage, is sufficient to yield impairments in the manipulation and rearrangement of information in working memory.

\section{Lesion laterality}

Because the majority of patients in the original SupPar Lesion group had unilateral lesions, we were able to examine the effect of lesion laterality on working memory performance by dividing the SupPar Lesion group into those patients with exclusively right hemisphere lesions $(n=9)$ and those with exclusively left hemisphere lesions ( $n=4$; supplemental Table 6 , available at www. jneurosci.org as supplemental material). The remaining six individuals had lesions that involved both cerebral hemispheres, although not necessarily involving the superior parietal cortex in both hemispheres. The only significant difference between the right and left hemisphere lesion groups was on Spatial Span Backward, where patients with right superior parietal lesions performed significantly worse than patients with left superior parietal lesions ( $p=0.03$ ). These results suggest that lesion laterality within SPL may be a critical factor for working memory involving visuospatial manipulation, but not in working memory test performance more generally. However, due to the exclusion of subjects with significant language impairment, most of whom had left hemisphere lesions, the relatively small number of left 


\begin{tabular}{|c|c|c|c|c|c|c|}
\hline & No Lesion & NonSupPar Lesions & SupPar Lesions & ANOVA Fvalue & ANOVA $p$ value & Significant between-group differences \\
\hline Logical Memory: Immediate recall & $39.9(8.3)$ & $37.6(9.1)$ & $34.5(7.5)$ & 2.9 & 0.06 & None \\
\hline Logical Memory: Delayed recall & $21.7(6.9)$ & $20.7(7.5)$ & $18.3(7.0)$ & 1.6 & 0.21 & None \\
\hline Logical Memory: Immediate - Delayed & $18.2(4.9)$ & $16.9(4.9)$ & $16.2(5.2)$ & 1.8 & 0.17 & None \\
\hline Faces: Immediate recognition & $34.5(4.5)$ & $32.5(4.7)$ & $32.1(5.3)$ & 3.8 & 0.02 & NonSupPar $<$ No Lesion \\
\hline Faces: Delayed recognition & $35.4(3.7)$ & $33.3(4.2)$ & $30.8(4.6)$ & 9.5 & $<0.001$ & SupPar $<$ NonSupPar $<$ No Lesion \\
\hline Faces: Immediate - Delayed & $-0.9(3.9)$ & $-0.8(4.4)$ & $1.3(4.1)$ & 2.1 & 0.12 & None \\
\hline Verbal-Paired Assoc: Immediate recall & $16.4(8.1)$ & $16.3(8.2)$ & $13.3(10.0)$ & 1.1 & 0.33 & None \\
\hline Verbal-Paired Assoc: Delayed recall & $5.2(2.4)$ & $4.9(2.5)$ & $3.8(3.0)$ & 1.9 & 0.15 & None \\
\hline Verbal-Paired Assoc: Immediate - Delayed & $11.3(6.3)$ & $11.4(6.3)$ & $9.5(7.8)$ & 0.8 & 0.47 & None \\
\hline Family Pictures: Immediate recall & $41.1(11.6)$ & $37.7(11.2)$ & $32.1(9.3)$ & 4.8 & 0.009 & SupPar $<$ No Lesion \\
\hline Family Pictures: Delayed recall & $40.3(12.1)$ & $36.9(11.8)$ & $31.3(8.9)$ & 4.3 & 0.01 & SupPar $<$ No Lesion \\
\hline Family Pictures: Immediate - Delayed & $0.8(3.8)$ & $0.8(5.1)$ & $0.7(4.4)$ & 0.001 & 0.99 & None \\
\hline
\end{tabular}

Means are presented with SDs in parentheses. One-way ANOVAs were conducted for each long-term memory test. Significant between-group differences were determined with Tukey's honestly significant difference test. Assoc, Associates.

Table 5. Rearrangement test data

\begin{tabular}{|c|c|c|c|c|c|c|}
\hline & No Lesion & NonSupPar Lesions & SupPar Lesions & ANOVA Fvalue & ANOVA $p$ value & Significant between-group differences \\
\hline Tower Test & $17.7(4.0)$ & $17.1(4.2)$ & $15.9(4.1)$ & 1.3 & 0.29 & None \\
\hline Block Design & $35.4(10.3)$ & $34.3(10.7)$ & $28.8(10.1)$ & 2.7 & 0.07 & None \\
\hline Object Assembly & $28.2(8.4)$ & $27.6(8.1)$ & $23.8(8.2)$ & 2.0 & 0.13 & None \\
\hline
\end{tabular}

Means are presented with SDs in parentheses. One-way ANOVAs were conducted for each test.

unilateral SPL lesions included in this study $(n=4)$ precludes any definitive conclusions about SPL lesion laterality.

\section{Specificity of cognitive deficit}

The working memory results, coupled with previous studies suggesting a role for parietal cortex in long-term memory processing (Wagner et al., 2005; Cabeza et al., 2008), raise the question of whether superior parietal lesions specifically impair working memory processes, or whether they impair more general encoding or retrieval processes that would also affect long-term memory. To answer this question, we compared the performance of the SupPar Lesion, NonSupPar Lesion, and No Lesion groups on four tests of long-term memory from the Wechsler Memory Scale-III (Wechsler, 1997b): Logical Memory, Faces, VerbalPaired Associates, and Family Pictures. The difference in scores between the "immediate" and "delayed" tests ("immediate delayed" score) is an index of long-term memory retention. Table 4 shows the group means for immediate, delayed, and immediate - delayed scores for each of the four tests. The SupPar Lesion group was not significantly different from either comparison group on the immediate - delayed scores for any of the four tests, nor was the SupPar Lesion group significantly different from either comparison group on the immediate and delayed conditions of Logical Memory and Verbal-Paired Associates, both of which have auditory-verbal stimuli. The SupPar Lesion group did score significantly lower than the No Lesion group on certain components of the tests with visual stimuli (specifically, the immediate and delayed recall for Family Pictures, as well as the delayed recognition for Faces), but the NonSupPar Lesion group also scored significantly lower than the No Lesion group on tests with visual stimuli (specifically, the immediate and delayed recognition for Faces). Unlike the working memory tests, where the SupPar Lesion group (and only the SupPar Lesion group) exhibited deficits in both auditory-verbal and visual-spatial domains, these long-term memory tests reveal no pervasive memory deficits specific to superior parietal damage.

Another possibility is that superior parietal damage may not only impair the manipulation and rearrangement of information in working memory, but rather, may impair rearrangement or reordering processes more broadly. To address this possibility, we compared the performance of the SupPar Lesion, NonSupPar Lesion, and No Lesion groups on three cognitive tests that require reordering or rearrangement processes, but not the retention of information in working memory: Tower Test, Block Design, and Object Assembly. Although the scores of the SupPar Lesion group were, on average, lower than the comparison groups' scores for each of these three tests, none of the differences reached statistical significance (Table 5). These data suggest that the effect of superior parietal lesions on mental rearrangement and manipulation may indeed be specific to working memory. However, we regard this conclusion as provisional due to the fact that the nonworking memory rearrangement tests are not necessarily equivalent to the working memory tests in terms of task difficulty or variance in performance. It is possible that the superior parietal lesion patients have broad manipulation/rearrangement deficits that are particularly revealed by tasks with an additional cognitive load, such as the mnemonic component of the working memory tests.

\section{Discussion}

In this study, we investigated whether SPL plays a critical role in human working memory. Using a relatively large sample of patients with superior parietal damage $(n=19)$ and a wide-ranging assessment of cognitive function, we report several main findings: (1) SPL lesions are associated with deficits in the manipulation and rearrangement of information within working memory, but not the simple retention and retrieval of information in working memory; (2) SPL lesions are associated with deficits in the manipulation and rearrangement of information within working memory for both auditory-verbal and visual-spatial stimuli; and (3) SPL lesions are not associated with pervasive deficits in tests of longterm memory or spatial rearrangement not involving working memory. Together, these results suggest that SPL is critically important for the manipulation and rearrangement of information in working memory.

One issue that is not directly addressed by our data is whether the observed cognitive deficits subsequent to SPL lesions are due to damage to the SPL gray matter, or whether the cognitive deficits instead arise from damage to the subjacent white matter 
pathways that link SPL with areas such as IPS or lateral frontal cortex. Indeed, most neurobiological models of working memory and executive function implicate a frontoparietal network (Chafee and Goldman-Rakic, 2000; Corbetta and Shulman, 2002), and clearly the SPL lesions in this study involve both gray and white matter damage (Fig. 1; supplemental Fig. 1, available at www.jneurosci.org as supplemental material). One can assume that in addition to the clearly delineated tissue loss in and beneath SPL, there would also likely be some degree of secondary degeneration of the affected white matter pathways that is undetectable with CT. Although the secondary white matter damage in our sample is likely comparable to that of ischemic stroke, subarachnoid hemorrhage, or surgical resection, we must certainly acknowledge that degenerative white matter damage may exacerbate the cognitive deficits in our patients. However, there are two lines of evidence that suggest widespread white matter damage, and more specifically frontoparietal tract damage, is not primarily responsible for the observed results. First is the comparable performance of SPL patients to the neurologically healthy vets on many of the cognitive tests (e.g., Digit Span Forward, 0-Back, 1-Back, Tower Test, language tests, and verbal long-term memory tests). This pattern of cognitive performance starkly contrasts that of patients with brain injuries involving diffuse white matter damage (such as traumatic brain injury), who typically exhibit deficits in attention, executive function, and long-term memory (Lezak et al., 2004). Second is the observation that individuals from the VHIS sample with dorsolateral PFC lesions $(n=21)$ exhibit no significant impairment (relative to the neurologically healthy comparison vets) on any tests of working memory retention or manipulation (Digit Span, Spatial Span, N-Back, Letter-Number Sequencing, or Arithmetic). (These data have been submitted as a separate manuscript.) Since these dorsolateral PFC lesions also intersect frontoparietal white matter pathways and most certainly disrupt frontal lobe function to a greater extent than the SPL lesions, we conclude that SPL lesions impair working memory manipulation through their effects within superior parietal cortex, rather than through downstream effects on frontal lobe.

Our findings are germane to several areas of research. One related area is functional neuroimaging research on the neural correlates of working memory. The neurological patient data reported here are consistent with a meta-analysis of dozens of functional imaging studies (Wager and Smith, 2003), which concludes that the superior parietal cortex is the region most commonly activated by tasks involving the ordering, updating, and manipulation of items in working memory. Accordingly, among the superior parietal lesion patients we reliably found deficits on tests requiring exactly those cognitive operations.

A second related area of research is the use of repetitive transcranial magnetic stimulation (rTMS) to modulate working memory performance. Several studies have reported that rTMS to PPC/SPL can affect the simple short-term retention as well as manipulation of information in working memory (Koch et al., 2005; Postle et al., 2006; Luber et al., 2007; Hamidi et al., 2008; Hamidi et al., 2009). These rTMS findings differ somewhat from our lesion findings in that tests of simple retention and recall were affected by rTMS to SPL, but not by focal damage involving SPL. One possible explanation for this apparent discrepancy is that the effects of rTMS are "acute," whereas the patients in our study acquired their lesions several decades before behavioral testing. Thus, it may be that although many aspects of working memory function are supported by the SPL in the healthy brain, these functions are differentially amenable to long-term compensatory reorganization that can follow traumatic injury. It is also the case, however, that caution is warranted in drawing structure-function inferences from rTMS studies, because some of the behavioral effects of rTMS may result from physiological effects on cortical regions that are distal to the region directly under the stimulating coil. For example, in one study that recorded the EEG while rTMS was delivered to SPL, the rTMS-related effects on the EEG that correlated with rTMS-related changes in spatial delayed-recognition performance were not source localized to the SPL, but rather to cortical regions in posterior frontal cortex and in parieto-occipital cortex (Hamidi et al., 2009). In this regard it is noteworthy that whereas damage in our SPL lesion group was largely restricted to the PPC, one might expect to find deficits in the simple retention of information in patients with more widespread cortical damage. The patients excluded due to significant language impairment inform this question to some degree. This group was characterized by widespread left hemisphere damage (involving multiple cortical lobes) as well as conspicuously low Digit Span Forward scores (mean 4.7, SD 1.1). This result lends qualified support to accounts of verbal working memory positing strong links between short-term phonological storage and receptive and/or productive components of language (Acheson and MacDonald, 2009).

A third related area of research is the role of PPC in visuospatial processing. Although we did not observe pervasive visuospatial processing impairments in the SPL lesion patients, one intriguing possibility is that the monitoring and manipulation of information in working memory is dependent on the neural substrates involved in the monitoring and manipulation of objects in visual space. In other words, the neural systems representing physical spatial arrangement have been adaptively co-opted to represent the mental arrangement of information in working memory. Consistent with this proposition are the results of at least two functional imaging studies (LaBar et al., 1999; Knops et al., 2009), which report that the same area of PPC can be activated during tasks of visuospatial attention as well as during ostensibly nonspatial tests involving working memory. Another possibility is that superior parietal cortex plays a more general, modalityindependent role in the allocation of attention. Functional imaging studies demonstrate that SPL is activated not only when subjects are cued to shift their visual attention from one location to another (Yantis et al., 2002; Molenberghs et al., 2007), but also when subjects are cued to shift their auditory attention from one location to another (Shomstein and Yantis, 2006), or their auditory attention from one voice to another (Shomstein and Yantis, 2006), or their attention between visual and auditory stimuli (Shomstein and Yantis, 2004). Perhaps the superior parietal cortex is critical for certain working memory operations by virtue of its underlying role in the flexible allocation of attention; the manipulation of information within working memory seemingly requires rapid shifts of attention between the original stimuli and the iteratively updated reconfigurations.

In conclusion, the present lesion study provides a causal link between brain and behavior, demonstrating that the superior parietal cortex is indeed necessary for the executive rearrangement of information in working memory. Although for decades the prefrontal cortex has been recognized as the de facto "seat" of executive function, the neurological patient results reported here indicate a prominent role for the posterior parietal cortex in the mental manipulation of information in working memory. 


\section{References}

Acheson DJ, MacDonald MC (2009) Verbal working memory and language production: common approaches to the serial ordering of verbal information. Psychol Bull 135:50-68.

Baldo JV, Dronkers NF (2006) The role of inferior parietal and inferior frontal cortex in working memory. Neuropsychology 20:529-538.

Berryhill ME, Olson IR (2008a) The right parietal lobe is critical for visual working memory. Neuropsychologia 46:1767-1774.

Berryhill ME, Olson IR (2008b) Is the posterior parietal lobe involved in working memory retrieval? Evidence from patients with bilateral parietal lobe damage. Neuropsychologia 46:1775-1786.

Boller F, Vignolo LA (1966) Latent sensory aphasia in hemisphere-damaged patients: an experimental study with the token test. Brain 89:815-830.

Cabeza R, Ciaramelli E, Olson IR, Moscovitch M (2008) The parietal cortex and episodic memory: an attentional account. Nat Rev Neurosci 9: 613-625.

Chafee MV, Goldman-Rakic PS (2000) Inactivation of parietal and prefrontal cortex reveals interdependence of neural activity during memoryguided saccades. J Neurophysiol 83:1550-1566.

Colby CL, Goldberg ME (1999) Space and attention in parietal cortex. Annu Rev Neurosci 22:319-349.

Collins DL, Neelin P, Peters TM, Evans AC (1994) Automatic 3D intersubject registration of MR volumetric data in standardized Talairach space. J Comput Assist Tomogr 18:192-205.

Corbetta M, Shulman GL (2002) Control of goal-directed and stimulusdriven attention in the brain. Nat Rev Neurosci 3:201-215.

Critchley M (1953) The parietal lobes. New York: Hafner.

Delis DC, Kaplan E, Kramer JH (2001) Delis-Kaplan Executive Function System (D-KEFS): examiner's manual. San Antonio, TX: The Psychological Corporation.

Goodglass H, Kaplan E (2000) Boston Naming Test. Philadelphia: Lippincott Williams and Wilkins.

Hamidi M, Tononi G, Postle BR (2008) Evaluating frontal and parietal contributions to spatial working memory with repetitive transcranial magnetic stimulation. Brain Res 1230:202-210.

Hamidi M, Slagter HA, Tononi G, Postle BR (2009) Repetitive transcranial magnetic stimulation affects behavior by biasing endogenous cortical oscillations. Front Integr Neurosci 3:14.

Husain M, Mannan S, Hodgson T, Wojciulik E, Driver J, Kennard C (2001) Impaired spatial working memory across saccades contributes to abnormal search in parietal neglect. Brain 124:941-952.

Knops A, Thirion B, Hubbard EM, Michel V, Dehaene S (2009) Recruitment of an area involved in eye movements during mental arithmetic. Science 324:1583-1585.

Koch G, Oliveri M, Torriero S, Carlesimo GA, Turriziani P, Caltagirone C (2005) rTMS evidence of different delay and decision processes in a fronto-parietal neuronal network activated during spatial working memory. Neuroimage 24:34-39.

LaBar KS, Gitelman DR, Parrish TB, Mesulam M (1999) Neuroanatomic overlap of working memory and spatial attention networks: a functional MRI comparison within subjects. Neuroimage 10:695-704.

Lezak MD, Howieson DB, Loring DW (2004) Neuropsychological assessment. New York: Oxford UP.

Luber B, Kinnunen LH, Rakitin BC, Ellsasser R, Stern Y, Lisanby SH (2007) Facilitation of performance in a working memory task with rTMS stimulation of the precuneus: frequency- and time-dependent effects. Brain Res 1128:120-129.

Makale M, Solomon J, Patronas NJ, Danek A, Butman JA, Grafman J (2002)
Quantification of brain lesions using interactive automated software. Behav Res Methods Instrum Comput 34:6-18.

Malhotra P, Jäger HR, Parton A, Greenwood R, Playford ED, Brown MM, Driver J, Husain M (2005) Spatial working memory capacity in unilateral neglect. Brain 128:424-435.

Molenberghs P, Mesulam MM, Peeters R, Vandenberghe RR (2007) Remapping attentional priorities: differential contribution of superior parietal lobule and intraparietal sulcus. Cereb Cortex 17:2703-2712.

Nachev P, Husain M (2006) Disorders of visual attention and the posterior parietal cortex. Cortex 42:766-773.

Pisella L, Berberovic N, Mattingley JB (2004) Impaired working memory for location but not for colour or shape in visual neglect: a comparison of parietal and non-parietal lesions. Cortex 40:379-390.

Postle BR, Ferrarelli F, Hamidi M, Feredoes E, Massimini M, Peterson M, Alexander A, Tononi G (2006) Repetitive transcranial magnetic stimulation dissociates working memory manipulation from retention functions in the prefrontal, but not posterior parietal, cortex. J Cogn Neurosci 18:1712-1722.

Ravizza SM, Behrmann M, Fiez JA (2005) Right parietal contributions to verbal working memory: spatial or executive? Neuropsychologia 43:20572067.

Sack AT (2009) Parietal cortex and spatial cognition. Behav Brain Res 202:153-161.

Saffran EM, Marin OS (1975) Immediate memory for word lists and sentences in a patient with deficient auditory short-term memory. Brain Lang 2:420-433.

Shomstein S, Yantis S (2004) Control of attention shifts between vision and audition in human cortex. J Neurosci 24:10702-10706.

Shomstein S, Yantis S (2006) Parietal cortex mediates voluntary control of spatial and nonspatial auditory attention. J Neurosci 26:435-439.

Solomon J, Raymont V, Braun A, Butman JA, Grafman J (2007) Userfriendly software for the analysis of brain lesions (ABLe). Comput Methods Programs Biomed 86:245-254.

Tzourio-Mazoyer N, Landeau B, Papathanassiou D, Crivello F, Etard O, Delcroix N, Mazoyer B, Joliot M (2002) Automated anatomical labeling of activations in SPM using a macroscopic anatomical parcellation of the MNI MRI single-subject brain. Neuroimage 15:273-289.

van Asselen M, Kessels RP, Neggers SF, Kappelle LJ, Frijns CJ, Postma A (2006) Brain areas involved in spatial working memory. Neuropsychologia 44:1185-1194.

Vandenberghe R, Gillebert CR (2009) Parcellation of parietal cortex: convergence between lesion-symptom mapping and mapping of the intact functioning brain. Behav Brain Res 199:171-182.

Wager TD, Smith EE (2003) Neuroimaging studies of working memory: a meta-analysis. Cogn Affect Behav Neurosci 3:255-274.

Wagner AD, Shannon BJ, Kahn I, Buckner RL (2005) Parietal lobe contributions to episodic memory retrieval. Trends Cogn Sci 9:445-453.

Warrington EK, Logue V, Pratt RT (1971) The anatomical localisation of selective impairment of auditory verbal short-term memory. Neuropsychologia 9:377-387.

Wechsler D (1997a) Wechsler Adult Intelligence Scale-III. San Antonio, TX: The Psychological Corporation.

Wechsler D (1997b) Wechsler Memory Scale, ed 3 manual. San Antonio, TX: The Psychological Corporation.

Yantis S, Schwarzbach J, Serences JT, Carlson RL, Steinmetz MA, Pekar JJ, Courtney SM (2002) Transient neural activity in human parietal cortex during spatial attention shifts. Nat Neurosci 5:995-1002. 